\title{
Was versteht man unter Medical Affairs?
}

Jean-Marc C. Häusler

Die Darstellung im vorliegenden Artikel ist die persönliche Meinung des Autors und widerspiegelt nicht notwendigerweise die Sichtweise des Arbeitgebers.

Korrespondenz:

Dr. med. Jean-Marc C. Häusler, MBA UCB, Inc.

Medical Director

1950 Lake Park Drive

USA-Smyrna, GA 30080

Tel. 0017709708541

jean-marc.haeusler@ucb-group.com
Ärztinnen und Ärzte in der Pharmaindustrie arbeiten oft in der klinischen Entwicklung oder in Medical Affairs. Medical Affairs ist mithin ein integraler Teil der pharmazeutischen Medizin und auch des FMH-Curriculums zum Facharzt für Pharmazeutische Medizin. Doch was versteht man eigentlich unter Medical Affairs? Was macht ein Medical Advisor bzw. was machen die Medical Science Liaisons? Das Ziel dieses Artikels ist es, den Begriff Medical Affairs und relevante Unterdisziplinen zu diskutieren und damit den praktizierenden Ärztinnen und Ärzten den Umgang mit Vertretern der Medical Affairs zu erleichtern.

\section{Die Wertschöpfungskette in der pharmazeutischen Industrie}

Die Wertschöpfungskette in der Pharmaindustrie kann man grob in zwei Bereiche unterteilen: die Entwicklung (bevor ein Medikament die Marktzulassung erhält) und die Vermarktung (nach Erteilung der Marktzulassung) (Abb. 1). Die Entwicklung besteht aus der Forschung nach neuen Substanzen, gefolgt von der präklinischen Entwicklung, wo im Labormodell und Tierversuch das Sicherheitsprofil und mögliche therapeutische Effekte genauer untersucht werden. In der klinischen Entwicklung werden hierauf interessante Substanzen bezüglich ihrer Pharmakologie, Sicherheit und Effektivität in mehreren Studien am Menschen getestet. Die Regulierungsbehörde prüft dann die Datenlage und erteilt gegebenfalls die Marktzulassung.

Die anschliessende Phase der Vermarktung besteht aus den Bereichen Marketing, Verkauf und Medical Affairs. Dies sind drei parallel ablaufende Prozesse. Das Marketing plant die Produktstrategie und stellt Werbe- und Verkaufsmaterial her. Der Verkauf besteht aus den Ärztebesucherinnen und -besuchern (auch Pharmaberater oder auf Neudeutsch Sales Representatives genannt). Medical Affairs schliesslich kümmert sich darum, neue Informationen über die vermarkteten Medikamente zu generieren und Personen im Gesundheitswesen zugänglich zu machen.

\section{Vom Medical Marketing zu Medical Affairs} Medical Affairs hat sich erst in den letzten zehn bis zwanzig Jahren als eigenständiger Bereich etabliert. Die meisten Funktionen wurden vorher hauptsächlich vom Marketing wahrgenommen und als Medical Marketing bezeichnet. Dabei trat

\section{Zusammenfassung}

Medical Affairs ist neben dem Marketing und dem Verkauf einer der drei Bereiche, der sich um die Vermarktung pharmazeutischer Produkte kümmert. Aufgrund regulatorischer Entwicklungen in den USA hat sich Medical Affairs im Verlauf der letzten zehn bis zwanzig Jahre als eigenständiger Bereich vom Marketing abgespalten. Medical Affairs befasst sich damit, neue Einsichten und neues Wissen um vermarktete Medikamente zu generieren und zu verbreiten. Neues Wissen wird generiert durch die Durchführung klinischer Studien der Phasen IIIb und IV. Wissensverbreitende Funktionen sind hauptsächlich das Publikationsmanagement, die Medizinische Information und der medizinische Aussendienst. Wissen wird auch intern weitergegeben, so ewa bei der Prüfung von Werbe- und Verkaufsmaterial und bei der Ausbildung der Ärztebesucher. Medical-AffairsAbteilungen werden oft als Matrix strukturiert mit Medical Advisors als produktspezifischen Projektmanagern und den verschiedenen Unterdisziplinen als Matrixfunktionen. Die Etablierung der Medical Affairs hat sich als wertvoll erwiesen, um Interessenkonflikte zu vermindern, die Spätphasenforschung zu fördern und das Management spezifisch medizinischer Inhalte wie etwa von Publikationen und medizinischer Information zu pro-fessionalisieren.

jedoch folgendes Dilemma auf: Für Medizinalpersonen sind manchmal Informationen über ein Medikament hilfreich, die nicht mit der Marketingstrategie übereinstimmen (so etwa wenn diese Informationen negative oder inkonklusive Daten betreffen) oder die von Marketing und Verkauf nicht bereitgestellt werden dürfen, da sie etwa eine nicht zugelassene Indikation oder ein Medikament in Entwicklung betreffen. Überdies sind medizinische Informationen nur hilfreich, wenn sie wahr, ausgewogen und nicht irreführend sind. 


\section{Abbildung 1}

Die Wertschöpfungskette in der pharmazeutischen Industrie.

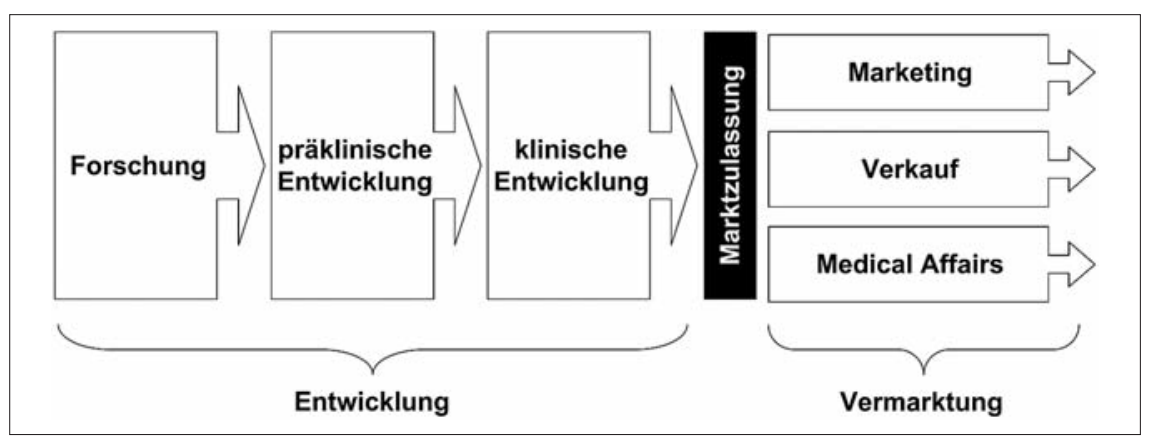

Je unabhängiger das Medical Marketing von kommerziellen Interessen ist, umso einfacher ist es, solche wissenschaftlichen Informationen anzubieten.

Die Abspaltung des Medical Marketing vom promotionellen Marketing wurde massgeblich durch die amerikanische Arzneimittelbehörde FDA mittels Gesetzen, Wegleitungsdokumenten und einigen spektakulären Prozessen wegen Vermarktung ausserhalb der zugelassenen Indikation (sogenanntes «off-label marketing») vorangetrieben. Deshalb wurden Marketing und Medical Affairs durch die meisten grösseren Pharmafirmen organisatorisch und finanziell getrennt, um damit juristisches Risiko und Haftung zu vermindern.

\section{Auftrag: Wissen}

Der Auftrag der Medical Affairs wird in jeder Firma etwas anders definiert. Ganz allgemein beinhaltet er aber meistens einige der folgenden Komponenten: das Generieren von neuen Einsichten und neuem Wissen über vermarktete oder bald zu vermarktende Produkte, das Erstellen von Beziehungen mit den verschiedenen Akteuren im Gesundheitswesen, die Verbreitung der Einsichten und Wissen an Ärztinnen und Ärzte und das Ziel, mit all diesen Aktivitäten die Behandlung der Patienten zu optimieren.

Die Mission im Falle des Autors beispielsweise lautet, Wissen zu generieren und zu benutzen, um Patientenergebnisse zu verbessern («generate and use knowledge to improve patient outcomes»). Hierbei ist beachtenswert, dass der Arzt in Medical Affairs sehr wohl auch die Salus Aegroti (das Wohl des Patienten) als Ziel vor Augen hat, wenn auch natürlich weniger direkt als der Arzt in der Praxis. Manchmal, besonders in kleineren Niederlassungen, werden die Arzneimittelsicherheit oder der Umgang mit Regulierungsbehörden (Regulatory Affairs) in die Medical Affairs integriert. Diese Bereiche gehören jedoch nicht zu den Kernfunktionen der Medical Affairs und werden hier nicht näher diskutiert.

\section{Wissen generieren und verbreiten}

Die wichtigste wissensgenerierende Funktion sind die Clinical Operations zur Durchführung klinischer Studien, hauptsächlich der Phasen IIIb und IV. Bei diesen Spätphasenstudien geht es nicht mehr darum, die Sicherheit und Effektivität eines Medikaments zu etablieren, sondern das Ziel ist es, das Medikament besser kennenzulernen, besonders im Vergleich mit anderen Therapien oder bezüglich spezieller Endpunkte wie etwa Lebensqualität oder Gesundheitsökonomie. Weitere Studien, die bisweilen durchgeführt werden, sind nichtinterventionelle Studien (Beobachtungsstudien) oder die Reanalyse existierender Daten (Data Mining).

Traditionell wird das hierbei generierte Wissen dann in Form von Publikationen weitergegeben. Das Publikationsmanagement umfasst dabei die Publikation firmeneigener Studien und unabhängiger Studien, sofern dies vom Studienleiter gewünscht wird, in Form von Kongressbeiträgen, primären Artikeln und manchmal Übersichtsartikeln. In jedem Fall wird diese Unterstützung in der Publikation selbst offengelegt.

Die Medizinische Information befasst sich damit, externe medizinische Anfragen mit kompletter wissenschaftlicher Information entweder am Telefon oder in schriftlicher Form zu beantworten. Hierfür werden proaktiv Standardbriefe vorbereitet, um die häufigsten Fragen kompetent diskutieren zu können. In manchen Fällen möchten Medizinalpersonen komplexe Fragen jedoch lieber in persönlichem Kontakt erörtern. Als Reaktion auf dieses Bedürfnis hat sich der medizinische Aussendienst entwickelt, die sogenannten MSL (Medical Science Liaisons) oder MSA (Medical Scientific Advisors). Diese sind mithin das «Gesicht» der medizinischen Information. Daneben können MSL auch bei der Studienplanung und -verwaltung mithelfen und wissenschaftliche Vorträge halten.

Als letzten Unterbereich gibt es noch die Medical Education, die sich mit der finanziellen Unterstützung der medizinischen Weiterbildung mittels sogenannter «Educational Grants» befasst. Hierbei werden Gesuche um finanzielle Unterstützung nach vorher festgelegten Kriterien beurteilt. Die sorgfältige Beurteilung der Programme ist wichtig, da die Pharmafirmen nach Erteilung einer finanziellen Unterstützung je nach Land wenig bis keinen Einfluss auf die Inhalte und die Qualität des Programms haben.

Wissen soll jedoch nicht nur extern, sondern auch innerhalb der Firma weitergegeben werden. Dazu gehören die fachliche Aus- und Weiterbildung der Ärztebesucher, aber auch medizinische Kommentare und Analysen an andere Abteilungen wie Marketing, klinische Entwicklung oder 


\section{Abbildung 2}

Mögliche Struktur einer Medical-Affairs-Abteilung: Der Medical Advisor als produktspezifischer Projektmanager in Zusammenarbeit mit den verschiedenen Matrixfunktionen.

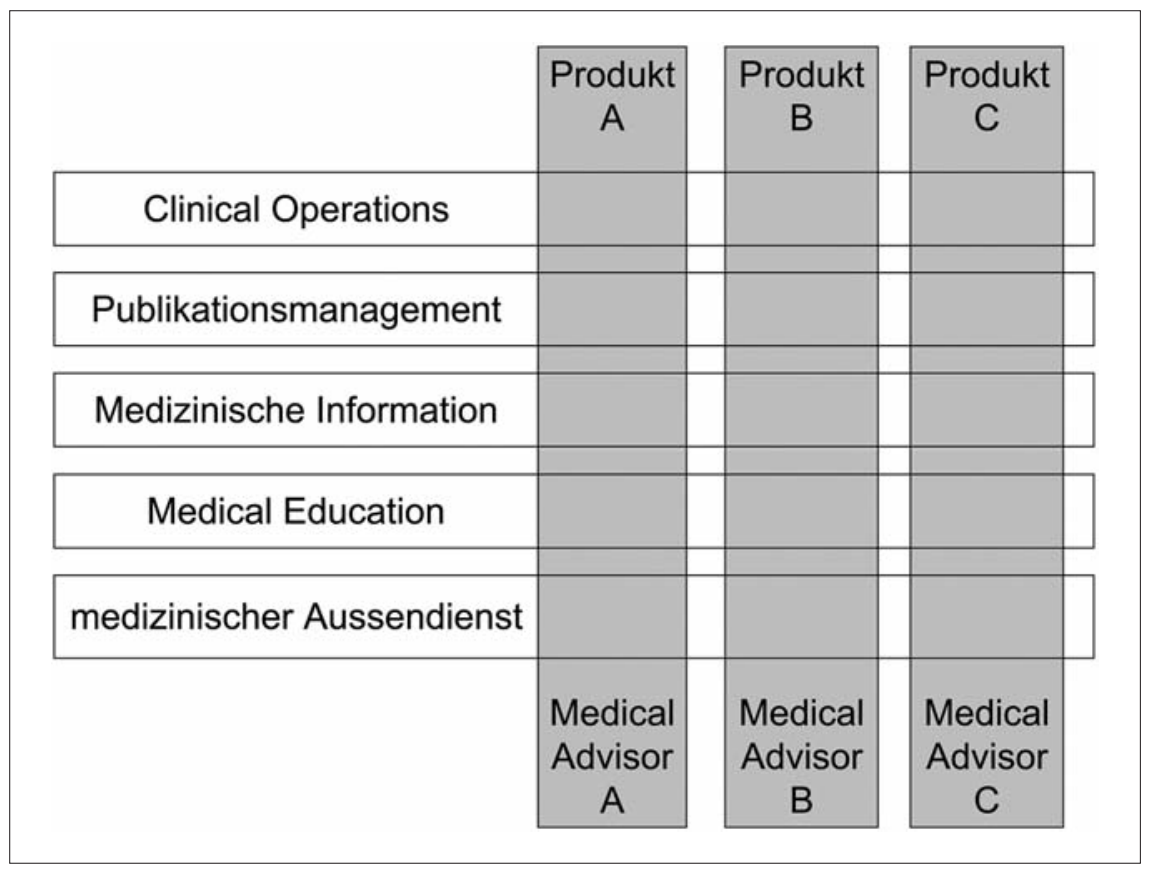

Regulatory Affairs. Medical Affairs ist schliesslich dafür verantwortlich sicherzustellen, dass alle Marketingaussagen wissenschaftlich korrekt, wahr, ausgewogen und nicht irreführend sind. Deshalb wird alles Werbe- und Verkaufsmaterial einer internen medizinischen Prüfung unterzogen, bevor es extern benutzt werden darf.

\section{Struktur einer Medical-Affairs-Abteilung}

Medical-Affairs-Abteilungen sind oft in einer Matrix organisiert und werden durch einen medizinischen Direktor geleitet (Abb. 2). Die produktspezifische Verantwortung wird durch mehrere Medical Advisors (auch Medical Manager genannt) wahrgenommen, die für ihre Produkte gewissermassen die Funktion eines Projektmanagers verrichten. Diese arbeiten zusammen mit den verschiedenen bereits besprochenen produktunspezifischen Matrixfunktionen. In grossen Niederlassungen, so etwa in den USA, werden diese Funktionen als eigene Unterabteilungen geführt; in kleineren können sie entweder durch einzelne Personen oder durch den Medical Advisor selbst wahrgenommen werden. Der medizinische Aussendienst ist typischerweise mit Verantwortung für alle Produkte in die Matrix integriert, kann aber auch in produktspezifischer Linienverantwortung einem Medical Advisor unterstellt sein.

Bedeutung der Medical-Affairs-Abteilung Medical Affairs als eigenständiger Bereich der Vermarktung und der pharmazeutischen Medizin ist noch jung und einer konstanten Entwicklung unterworfen. Nichtsdestotrotz hat sich die Etablierung der Medical Affairs als wertvoll erwiesen:

- Die Weitergabe von Wissen hat sich von einem Mittel für kommerzielle Ziele zu einem Zweck an sich entwickelt. Die damit reduzierten Interessenkonflikte mit dem Marketing helfen mit, das juristische Risiko von Pharmafirmen zu mindern.

- Der stärkere Fokus auf Spätphasenstudien ist nützlich, um mehr vergleichende Daten zu generieren und auch gesundheitsökonomische und andere Endpunkte anzugehen, die in der klinischen Entwicklung eines Medikaments üblicherweise nicht untersucht werden.

- Funktionen wie das Publikationsmanagement, die Medizinische Information, die Medical Education und auch die interne Prüfung von Werbe- und Verkaufsmaterial wurden professionalisiert, was die Qualität verbessert und konsistenter gemacht hat.

- Mit dem medizinischen Aussendienst haben Ärztinnen und Ärzte kompetente Ansprechspersonen, um komplexe wissenschaftliche Fragen zu diskutieren. 